\title{
Primary Subcutaneous Leiomyosarcoma of the Hamster Hind Leg
}

\author{
Jung-Yeon $\mathrm{YI}^{1)}$, Yeong-Hun $\mathrm{KIM}^{1)}$ and Byung-Il YOON ${ }^{1) *}$ \\ ${ }^{1)}$ School of Veterinary Medicine and Institute of Veterinary Science, Kangwon National University, 192-1 Hyoja-dong, Chuncheon, \\ Gangwon 200-701, Republic of Korea
}

(Received 16 October 2007/Accepted 9 January 2008)

ABSTRACT. We report the first case of a primary subcutaneous leiomyosarcoma that originated in the hind leg of a hamster and metastasized to the bone marrow, lung and diaphragm. A 10-month-old female Syrian golden hamster was presented with a large, firm, white subcutaneous mass in the right hind leg. The tumor invaded into the bone marrow and small nodules were also present in the lung and diaphragm; however, no tumor masses were found in the visceral organs. Histologically, the tumors were spindle cell sarcomas, composed of densely packed pleomorphic spindle cells with oval to elongate nuclei and moderate amounts of eosinophilic cytoplasm. Immunohistochemically, the neoplastic cells were positive for vimentin, desmin, and smooth muscle actin, but negative for $\alpha$-sarcomeric actin and S-100. Thus, the diagnosis was primary leiomyosarcoma of the hind leg with metastasis to the bone marrow, lung and diaphragm. To the best of our knowledge, this is the first report of spontaneous primary subcutaneous leiomyosarcoma of the hind leg with distant metastasis in laboratory animals.

KEY WORDS: hamster, hind leg, subcutaneous leiomyosarcoma.

Leiomyosarcoma is a malignant tumor of smooth muscle origin, characterized by broad interlacing fascicles of spindle-shaped neoplastic cells with elongate nuclei and eosinophilic cytoplasm. Leiomyosarcoma also shows malignant phenotypes such as pleomorphism, invasion, a relatively high mitotic index, necrosis, and metastasis [6]. This smooth muscle cell tumor may occur at any site in the body, because smooth muscle cells are widely distributed in every tissue and organ. Visceral organs, especially the gastrointestinal tract, spleen, and genital tract, have been shown to be relatively common sites for spontaneous leiomyosarcoma in domestic and experimental animals $[5,10,12,13$, $15,18,21]$. Leiomyosarcoma rarely occurs in other organs; only a few cases of leiomyosarcoma in the oral cavity, esophagus, and urinary bladder have been reported in domestic animals and mice $[4,6,11,15,17]$.

Cutaneous or subcutaneous leiomyosarcoma is extremely rare in animals. Although it has been reported infrequently in dogs, cats, ferrets, and monkeys [2, 3, 6, 14, 16], there have been no reports in laboratory animals. The cutaneous or subcutaneous development of this tumor usually occurs on the head or neck as a solitary dermal mass in animals, and metastasis is uncommon $[6,14]$.

In the present study, we describe a case of primary leiomyosarcoma in the hind leg of a hamster, which metastasized to the bone marrow, lung and diaphragm.

The 10-month-old female Syrian golden hamster presented in this study was housed at the animal facility of Kangwon National University and had been used for breeding. The hamster exhibited ataxia and behavioral coordination failure as a result of a large mass in the right hind leg.

\footnotetext{
* Correspondence to: Yoon, B. I., School of Veterinary Medicine, Kangwon National University, 192-1 Hyoja-dong, Chuncheon, Gangwon 200-701, Republic of Korea.

e-mail: byoon@kangwon.ac.kr
}

The hamster was sacrificed under ether anesthesia, and necropsy revealed a firm, whitish, large mass, measuring 50 $\times 50 \mathrm{~mm}$, in the subcutaneous region of the hind leg, surrounding the fractured long bone with whitish distinguishable nodules in the bone marrow cavity (Fig. 1A and 1B). Additionally, thickened, irregular and whitish areas were observed in the diaphragm (Fig. 1C). Multiple well-demarcated whitish nodules, approximately $2 \times 2 \mathrm{~mm}$ in size, were also present on the surface of the lung (Fig. 1D). The cut surface of the mass in the hind leg was yellowish-white, multinodular, and relatively avascular (Fig. 1A). No tumors were found in the visceral organs, including the gastrointestinal and genital tracts.
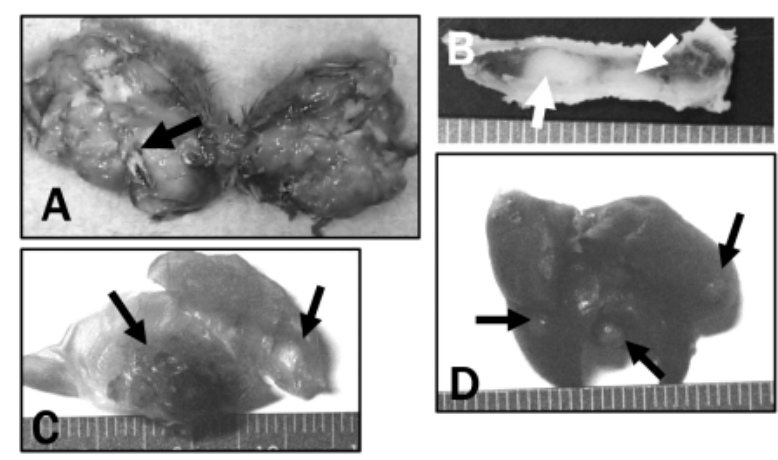

Fig. 1. Tumor gross findings. A large, firm and whitish mass, measuring $50 \times 50 \mathrm{~mm}$, was found in the subcutis of the right hind leg of hamster, surrounding a fractured long bone (arrow in A). The cut surface of the mass in the hind leg was yellowish-white, multinodular and relatively avascular (A). Also, note the irregularly demarcated whitish areas and nodules in the bone marrow (arrows in B) and diaphragm (arrows in C) and the multiple whitish nodules scattered on the surfaces of pulmonary lobes (arrows in D). Pictures B, C and D were taken after fixation in $10 \%$ buffered formalin. 
For histological examination, the masses and nodules were fixed in $10 \%$ neutral-buffered formalin, and tissue sections were prepared for hematoxylin and eosin staining. For the differential diagnosis, the sections were immunohistochemically stained for vimentin (monoclonal mouse antivimentin, Clone vim 3B4; DAKO Inc., Carpinteria, CA, USA; 1:100), desmin (mouse anti-human desmin, Clone 33; Biogenex, San Ramon, CA, U.S.A.; 1:100), smooth muscle actin (monoclonal mouse anti-human smooth muscle actin, Clone 1A4; DAKO; 1:100), and $\alpha$-sarcomeric actin (monoclonal mouse anti-sarcomeric actin, Clone Alpha-Sr-1; DAKO; 1:100), S-100 (rabbit polyclonal anti-S100; DAKO; $1: 1000)$ using the avidin-biotin complex method according to the manufacturer's instructions.

Histologically, the tumor in the hind leg was a nonencapsulated invasive sarcoma in the subcutis and was composed of densely packed pleomorphic spindle and round to ovoid cells with indistinguishable cellular borders (Fig. 2A). The neoplastic cells formed short, narrow interlacing fascicles, which invaded the skeletal muscle fibers of the subcutis.
The nuclei of the neoplastic cells were generally round to elongate or cigar-shaped, depending on the tissue section, and were extremely pleomorphic in shape and size with granular or dispersed chromatin (Fig. 2A). Megakaryotic and binucleate bizarre cells were barely evident (Inset of Fig. 2A). Areas of tumor necrosis containing vacuolated and edematous neoplastic cells were common. Mitotic figures were rarely found, with an occurrence ranging from zero to two at $\times 400$ magnification. Lymphocytic inflammatory cells were diffusely infiltrated among the tumor cells with mild mastocytic infiltration. The metastatic tumors in the bone marrow and the diaphragm and on the pleura of the lung were similar in morphology to those of the hind leg (Fig. 2B, 2C and 2D). Multiple embolic tumor masses were also evident in the parenchyma of the lung (Fig. 2C). In the diaphragm, neoplastic cells were invading into the adjacent normal tissue and had intersected the normal skeletal muscle fibers (Fig. 2D).

The immunohistochemical analysis showed that most of the neoplastic cells in the hind leg, bone marrow, diaphragm
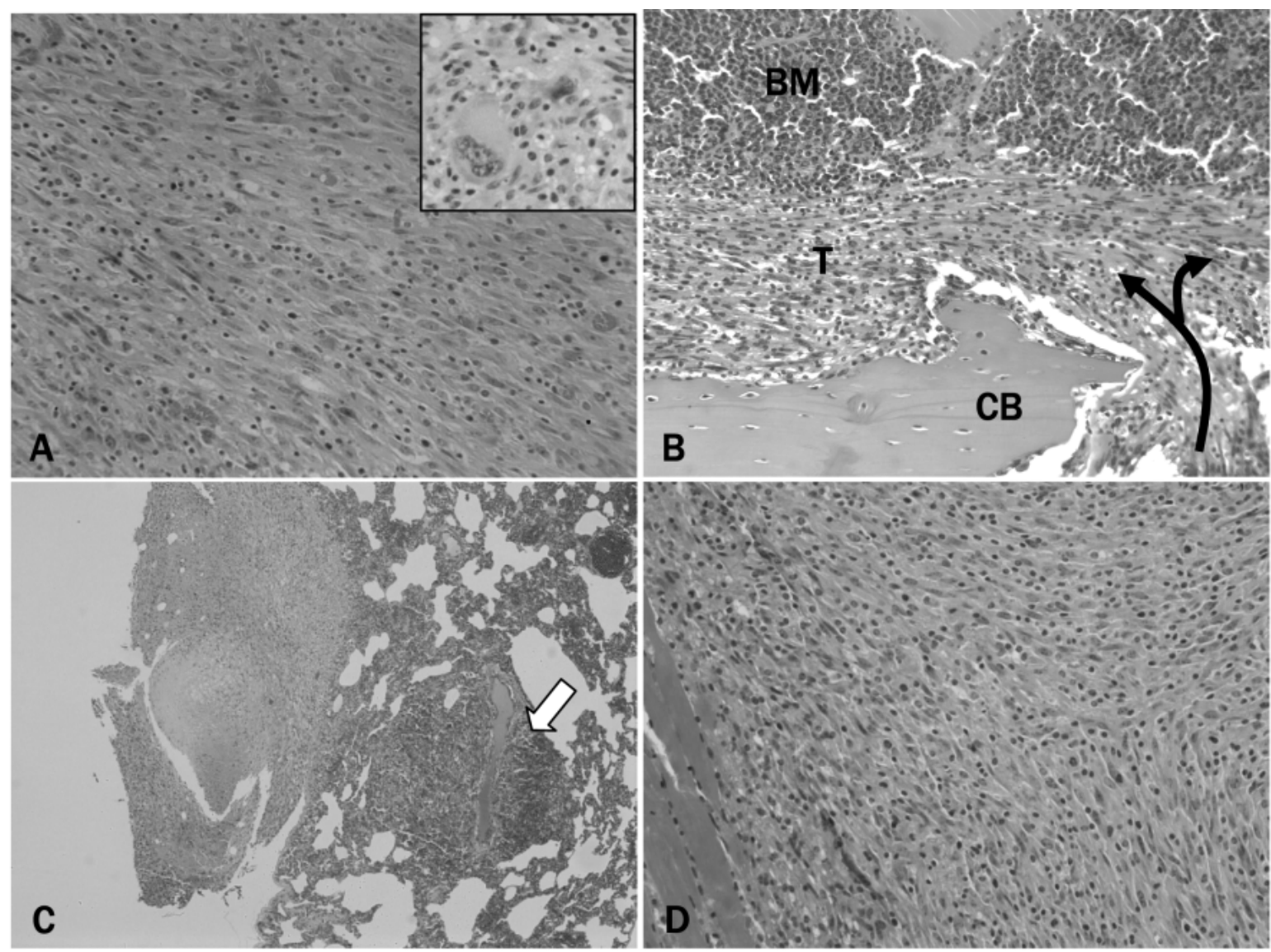

Fig. 2. Tumor histopathology. Note the spindle-shaped neoplastic cells with indistinguishable cellular borders and elongate, bluntended, and sometimes cigar-shaped nuclei, arranged in interlacing fascicles (A). Megakaryotic and binucleate bizarre cells were rarely evident (Inset of Fig. 2A). The neoplastic cells (T) invaded into the bone marrow (BM) through the compact bone (CB) of femur (arrows in B). The metastatic tumors in the bone marrow, pulmonary pleura and diaphragm had morphology similar to that of the hind leg tumor (B, C and D). Note the metastatic neoplastic foci in the parenchyma of the lung (white arrows in C). H\&E. Magnification: $\times 400$, except $\times 200$ in C. 


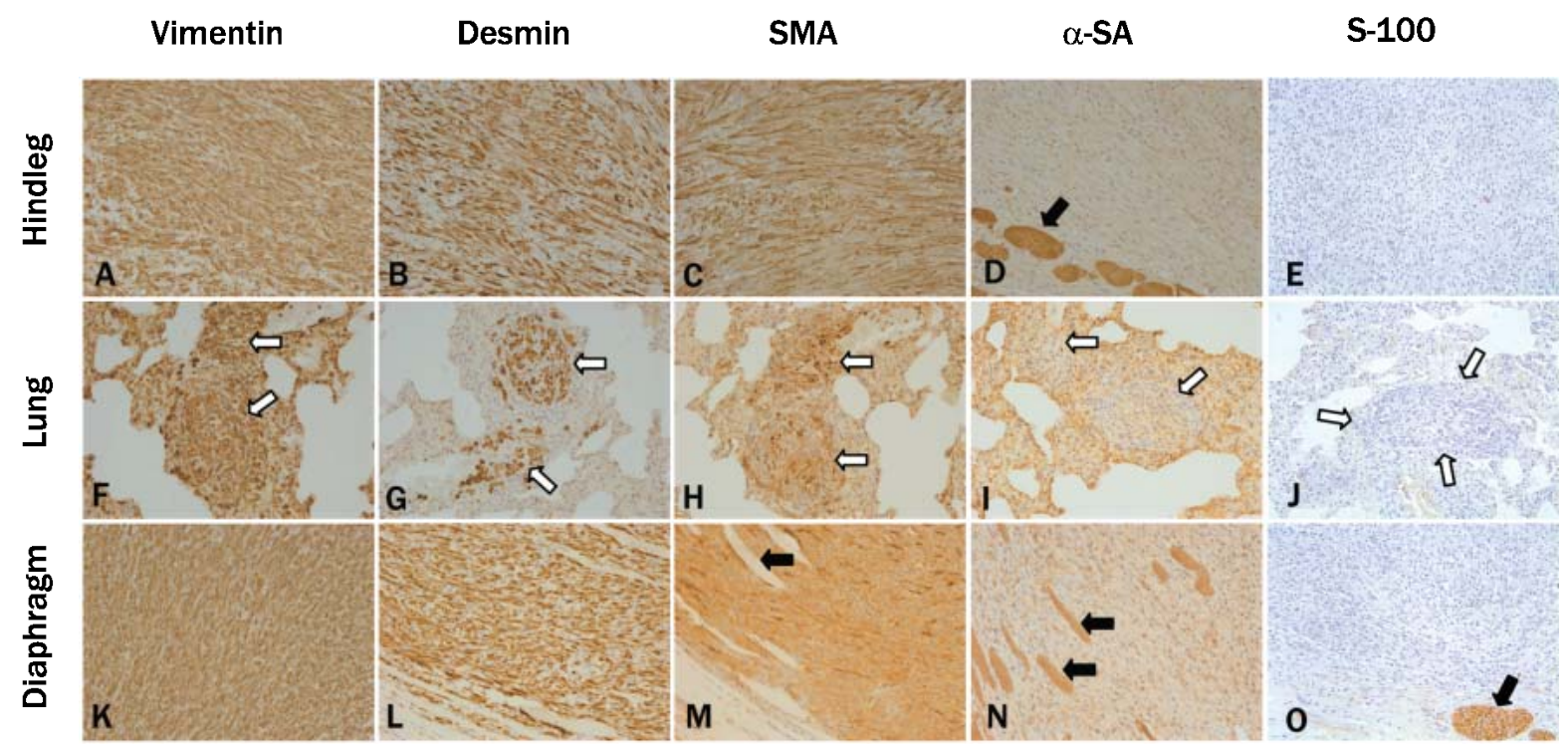

Fig. 3. Immunohistochemistry in the tumors. Both primary and metastatic neoplastic cells were consistently positive for vimentin (A, arrows in F, K), desmin (B, arrows in G, L), and smooth muscle actin (SMA) (C, arrows in H, M) but negative for $\alpha$-sarcomeric actin $(\alpha-S A)(D$, arrows in I, N) and S-100 (E, arrows in J, O). Note the contrasting immunohistochemical findings for SMA and $\alpha-S A$ in normal skeletal muscle fibers (arrows in D, M and N) and for S-100 in the nerve fibers in the diaphragm (arrow in O). Magnification: $\times 400$.

and lung were consistently positive for vimentin, desmin, and smooth muscle actin (SMA), but negative for $\alpha$-sarcomeric actin ( $\alpha$-SA) and S-100 (Fig. 3). The expression patterns of these proteins based on the antibody reactivities were comparable to those in the surrounding normal skeletal muscle (Fig. 3D, 3M and 3N). As no tumor masses were found in the most common sites of leiomyosarcoma $[6,13$, $15]$, i.e., the visceral organs or uterus, the hind leg was determined to be the primary tumor site.

Following the gross and histological examinations, the initial differential diagnoses were fibrosarcoma, rhabdomyosarcoma, synovial cell sarcoma, malignant schwannoma, osteosarcoma, and leiomyosarcoma. These diagnoses were based on the location of the tumor and the spindle-shaped, vimentin-positive neoplastic cells. Osteo- and chondrosarcoma were ruled out because we could not locate an osteoid or chondroid matrix [6] and no tumorigenic cellular alteration was observed in the bone tissue (Fig. 2B). Synovial cell sarcoma was also eliminated, based on a lack of morphological evidence associated with this tumor type, including synovioblastic cells, cavities, or slit-like spaces containing proteinaceous fluid or mucoid material. Furthermore, prominent nucleoli and a myxoid matrix were not observed [6]. Although malignant schwannoma could not be completely ruled out, it was not likely based on the histological absence of cellular sheets arranged in concentric whorls or a herringbone pattern [6]. In differentiating fibrosarcoma, schwannoma, rhadbomyosarcoma, and leiomyosarcoma, immunohistochemistry was used to determine the tumor cell origin. Both primary and metastatic neoplastic cells consistently showed strong positive immunoreactions for smooth muscle-specific actin and desmin but no positive reaction to the striated muscle-specific actin and S-100 antibodies. These results were consistent with previous descriptions of smooth muscle tumor cells $[5,6,11,13,21]$. Therefore, the diagnosis in the hamster was primary leiomyosarcoma of the hind leg with metastasis to the bone marrow, lung and diaphragm.

Very few cases of spontaneous leiomyosarcoma have been reported in hamsters, with reports of only one case each in the uterus and endometrium $[1,13]$. In addition, there are no previous reports of cutaneous leiomyosarcoma in laboratory animals, including hamsters. Reports of smooth muscle tumors of the skin and subcutis are also extremely rare in domestic animals, and most of those few tumors were benign $[6,14,16]$. To date, only a small number of cutaneous or subcutaneous leiomyosarcoma cases have been documented in dogs, cats, ferrets, cows, and monkeys $[2,3,6,8,14]$. Furthermore, unlike gastrointestinal leiomyosarcoma which metastasizes relatively frequently (in 7 to $33 \%$ of cases in dogs), subcutaneous tumors have rarely been observed to metastasize [5]. In our hamster case, subcutaneous leiomyosarcoma cells had metastasized from the hind leg to the bone marrow, diaphragm and lung. The metastasis of this case may be first achieved by invasion into the bone marrow, forming tumor emboli to move to the distant organs. Otherwise, as suggested in the previous studies [9, 14], if smooth muscle of blood vessel in subcutis were origin of this invasive malignant tumor, it might also be highly possible to form tumor emboli. Tumor emboli were evident in the blood vessels of the lung. Another possibility we could not rule out with regard to the tumor cell 
origin is from blood vessel in the bone marrow of the long bone, as infrequently reported in humans $[7,10,19,20]$, but it was unlikely in the present case on the basis of the metastatic nodules in the bone marrow.

Taken together, the gross, histological, and immunohistochemical findings strongly support a diagnosis of primary subcutaneous leiomyosarcoma of the hind leg which metastasized to the bone marrow, lung and diaphragm. This is the first report of primary subcutaneous leiomyosarcoma in the hind leg with distant metastasis in laboratory animals.

ACKNOWLEDGEMENTS. The authors thank Ms. NangIm Kim for her excellent assistance in this study. We also thank Dr. Dae-Yong Kim at Seoul National University for providing us with the antibodies used for immunohistochemistry. This study was supported by the Korea Research Foundation Grant (KRF-2006-003-E00346) and partially by Institute of Veterinary Science of Kangwon National University.

\section{REFERENCES}

1. Brownstein, D.G. and Brooks, A.L. 1980. J. Natl. Cancer Inst. 64: 1209-1214.

2. Brunnert, S.R., Herron, A.J. and Altman, N.H. 1990. Lab. Anim. Sci. 40: 208-210.

3. Brunnert, S.R., Herron, A.J. and Altman, N.H. 1990. Vet. Pathol. 27: 126-128.

4. Chandra, M. and Frith, C.H. 1991. Toxicol. Pathol. 19: 164167.

5. Cook, A.L., Rogers, T.D. and Sowers, M. 2004. Contemp. Top. Lab. Anim. Sci. 43: 47-49.

6. Cooper, B.J. and Valentine, B.A. 2002. pp. 319-363. In: Tumors in Domestic Animals, 4th ed. (Meuten D.J. ed.),
Blackwell Publishing, Iowa.

7. Goto, T., Ishida T., Motoi, N., Yokokura, S., Kawano, H., Yamamoto, A., Matsuda, K. and Yamamoto, M. 2002. J. Orthop. Sci. 7: 267-273.

8. Hanzaike, T., Ito, I., Ishikawa, T. and Kadota, K. 1995. J. Comp. Pathol. 112: 237-242.

9. Jacobsen, M.C. and Valentine, B.A. 2000. Vet. Pathol. 37: 100-103.

10. Jundt, G., Moll, C., Nidecker, A., Schilt, R. and Remagen, W. 1994. Hum. Pathol. 25: 1205-1212.

11. Kang, B.C., Jang, D.D. and Lee, S.K. 2005. J. Vet. Med. Sci. 67: 353-355.

12. Kapatkin, A.S., Mullen, H.S., Matthiesen, D.T. and Patnaik, A.K. 1992. J. Amer. Vet. Med. Assoc. 201: 1077-1079.

13. Kondo, H., Kimoto, H., Shibuya, H., Shirai, W., Matsuo, K. and Sato, T. 2007. J. Vet. Med. A. Physiol. Pathol. Clin. Med. 54: 27-29.

14. Liu, S.M. and Mikaelian, I. 2003. Vet. Pathol. 40: 685-692.

15. Maita, K., Hirano, M., Harada, T., Mitsumori, K., Yoshida, A., Takahashi, K., Nakashima, N., Kitazawa, T., Enomoto, A., Inui, K., et al. 1988. Toxicol. Pathol. 16: 340-349.

16. Mikaelian, I. and Garner, M.M. 2002. J. Vet. Diagn. Invest. 14: 262-265.

17. Norris, A.M., Laing, E.J., Valli, V.E.O., Withrow, S.J., Macy, D.W., Ogilvie, G.K., Tomlinson, J., McCaw, D., Pidgeon, G. and Jacobs, R.M. 1992. J. Vet. Int. Med. 6: 145-153.

18. Patnaik, A.K., Hurvitz, A.I. and Johnson, G.F. 1977. Vet. Pathol. 14: 547-555.

19. Shen, S.H., Steinbach, L.S., Wang, S.F., Chen, W.Y., Chen, W.M. and Chang, C.Y. 2001. Skeletal. Radiol. 30: 600-603.

20. Sundaram, M., Akduman, I., White, L.M., McDonald, D.J., Kandel, R. and Janney, C. 1999. AJR Am. J. Roentgenol. 172: 771-776.

21. Vemireddi, V., Langohr, I.M. and Thacker, H.L. 2007. J. Vet. Diagn. Invest. 10: 309-312. 